\title{
C-terminal binding protein-2 mediates cisplatin chemoresistance in esophageal cancer cells via the inhibition of apoptosis
}

\author{
HUI SHI ${ }^{1,2}$, YINTING MAO ${ }^{2}$, QIANQIAN JU ${ }^{2}$, YINGCHENG WU $^{3}$, WEN BAI $^{3}$, \\ PEIWEN WANG ${ }^{3}$, YUDONG ZHANG $^{2}$ and MAORONG JIANG ${ }^{1}$ \\ ${ }^{1}$ Laboratory Animals Center, Nantong University; ${ }^{2}$ Department of Thoracic Surgery, \\ Affiliated Hospital of Nantong University; ${ }^{3}$ Medical College of Nantong University, \\ Nantong, Jiangsu 226001, P.R. China
}

Received October 18, 2017; Accepted March 9, 2018

DOI: $10.3892 /$ ijo.2018.4367

\begin{abstract}
C-terminal binding protein-2 (CtBP2) is a transcriptional co-repressor that is associated with tumorigenesis and tumor progression. It has been reported to predict a poor prognosis in several human cancers, including esophageal squamous cell carcinoma (ESCC). The present study aimed to investigate the involvement of CtBP2 in the cisplatin (DDP) resistance of the ECA109 ESCC cell line and its effect on the expression of apoptosis-associated proteins. Constructed recombinant lentiviruses were used for the knockdown or overexpression of CtBP2 in ECA109 cells, and the expression of CtBP2 was measured using reverse transcription-quantitative polymerase chain reaction and western blotting following transfection. MTT assays, Hoechst 33342 staining and flow cytometry (FCM) were applied to detect the influence of CtBP2 on the DDP-induced viability and apoptosis of the transfected ECA109 cells. In addition, the levels of apoptosisassociated proteins, including p53, B-cell lymphoma 2 (Bcl-2), Bcl-2-associated X protein (Bax) and activated caspase-3 were investigated in the transfected ECA109 cells. Stable ECA109 cells with CtBP2 overexpression or knockdown were successfully established. The results of the MTT, Hoechst 33342 and FCM assays demonstrated that overexpression of CtBP2 attenuated the reduction of cell viability and inhibited the cell apoptosis induced by DDP. Furthermore, the western blotting results indicated that $\mathrm{CtBP} 2$ overexpression inhibited the DDP-induced apoptosis of ECA109 cells via the reduction of
\end{abstract}

Correspondence to: Professor Maorong Jiang, Laboratory Animals Center, Nantong University, 19 Qixiu Road, Nantong, Jiangsu 226001, P.R. China

E-mail: jiangmr@ntu.edu.cn

Professor Yudong Zhang, Department of Thoracic Surgery, Affiliated Hospital of Nantong University, 20 Xisi Road, Nantong, Jiangsu 226001, P.R. China

E-mail: mdzhangyd@126.com

Key words: C-terminal binding protein-2, esophageal squamous cell carcinoma, apoptosis, chemoresistance, cisplatin p53, activated caspase-3 and Bax expression, and promotion of $\mathrm{Bcl}-2$ expression. Therefore, the present study indicated that CtBP2 reduced the susceptibility of ECA109 cells to DDP by regulating the expression of apoptosis-related proteins, suggesting that it may be a promising therapeutic target in ESCC in the future.

\section{Introduction}

Esophageal cancer has a high morbidity rate and is the sixth most common cause of cancer-related mortality worldwide (1-3). A survey published in 2013 reported that there were 450,000 new cases of esophageal cancer annually (4), and China accounts for more than half of all cases of this type of cancer (5). Each year, it is estimated that 150,000 individuals succumb to esophageal cancer in China, and the 5-year survival rate of patients is usually $<30 \%(6-8)$. Esophageal cancer has two main subtypes, namely esophageal squamous cell carcinoma (ESCC) and esophageal adenocarcinoma $(9,10)$. Currently, the most commonly used treatments for ESCC are surgery, chemotherapy, radiation therapy and comprehensive treatment $(11,12)$. The preferred treatment for ESCC is surgical resection, but postoperative recurrence and distant metastasis are clinical problems for which effective treatments remain to be identified (13). Cisplatin (DDP) is a first-line drug in the treatment for ESCC, and the development of DDP resistance in ESCC cells is the main cause of chemotherapy failure $(14,15)$. The effectiveness of chemotherapy depends on the sensitivity of the tumor cells to chemotherapy drugs (16), and ESCC usually exhibits a high resistance to chemotherapy $(17,18)$. Therefore, the identification of oncogenes that may be targeted to combat resistance is likely to be a great benefit to clinical practice.

C-terminal binding protein 2 (CtBP2) acts as a transcriptional co-repressor, and modulates certain essential cellular processes, such as proliferation, migration and (19). It has been reported that $\mathrm{CtBP} 2$ has a critical function in tumorigenesis and tumor progression $(20,21)$. CtBP2 is overexpressed in a number of different tumor types, including hepatocellular carcinoma (22), prostate cancer $(23)$, breast cancer $(24,25)$ and ovarian cancer (26). Furthermore, preliminary studies conducted by the present research team revealed that the 
expression of CtBP2 was upregulated in ESCC tissues $(27,28)$. In addition, CtBP2 predicts a poor prognosis in human cancers, including ESCC (27). Therefore, it appears that CtBP2 serves an oncogene-like role in tumorigenesis and tumor progression. It has been reported that CtBP2 represses the sensitivity of breast cancer cells by p53-dependent and -independent mechanisms (29). However, the involvement of CtBP2 in the drug resistance of ESCC remains unknown.

In order to further understand the DDP resistance mechanisms of ESCC, the present study investigated the effect of CtBP2 on DDP resistance in ECA109 cells. To the best of our knowledge, the present study is the first to investigate this. The effect of knocking down CtBP2 on the susceptibility of ESCC cells to DDP was evaluated, and the underlying mechanism, such as the regulation of the expression of apoptosis-related proteins, was investigated. The results may indicate the potential of CtBP2 as a therapeutic target for ESCC.

\section{Materials and methods}

Cell culture. The human ESCC cell line ECA109 was supplied by the Cell Resource Center of Shanghai Institute for Biological Sciences, Chinese Academy of Sciences (Shanghai, China). Cell culture was performed in Dulbecco's modified Eagle's medium (DMEM; Thermo Fisher Scientific, Inc., Waltham, MA, USA) containing 10\% fetal calf serum (FCS; Hyclone; GE Healthcare Life Sciences, Logan, UT, USA), and $10 \mathrm{kU} / \mathrm{ml}$ penicillin and $10 \mathrm{mg} / \mathrm{ml}$ streptomycin (Beyotime Institute of Biotechnology, Haimen, China).

Knockdown or overexpression of $C t B P 2$. Firstly, recombinant lentiviral vectors were constructed to knockdown CtBP2 (LV-CtBP2-RNAi) or overexpress CtBP2 (LV-CtBP2). The sequences were designed using the software EPtiRNA (http://optirna.unl.edu/) and synthesized by GeneChem Co., Ltd. (Shanghai, China). The vector construction and virus packaging were conducted by GeneChem Co., Ltd. For the former, small hairpin RNA (shRNA) of CtBP2, whose target sequence was 5'-GCGCCTTGGTCAGTAATAG-3', was cloned into a GV248 vector (GeneChem Co., Ltd.) via EcoRI and AgeI restriction endonuclease sites. For the latter, the coding sequence of $\mathrm{CtBP} 2$ was cloned into a GV492 vector (GeneChem Co., Ltd.) via BamHI and AgeI restriction endonuclease sites. The primers used for overexpression were as follows: 5'-AGG TCG ACT CTA GAG GAT CCC GCC ACC ATG GCC CTT GTG GAT AAG CAC-3' (forward) and 5'-TCC TTG TAG TCC ATA CCT TGC TCG TTG GGG TGC TCT CGA TTG-3' (reverse). Schematic diagrams of the recombinant lentiviral vector constructs are presented in Fig. 1A.

ECA109 cells were transfected with LV-CtBP2-RNAi or LV-CtBP2 to knockdown or overexpress CtBP2, respectively. ECA109 cells transfected with empty vector GV248 and empty vector GV492 served as the negative controls, LV-CtBP2-RNAi and LV-CtBP2', respectively. The following formula was used to calculate to volume of virus to be added: Virus volume $=$ multiplicity of infection $\mathrm{x}$ cell number/virus titer. The cells $\left(1 \times 10^{6}\right.$ cells/well $)$ were seeded into 6 -well plates prior to transfection. In order to improve the transfection rate, $5 \mu \mathrm{g} / \mathrm{ml}$ polybrene and enhanced infection solution (without FCS) were incubated overnight with the cells at $37^{\circ} \mathrm{C}$ with $5 \% \mathrm{CO}_{2}$. Puromycin $(2 \mu \mathrm{g} / \mathrm{ml}$; Gibco; Thermo Fisher Scientific, Inc.) was added to the culture medium (DMEM containing $10 \%$ FCS) on the third day and refreshed every 2 days for 1 week to select the transfected cells. The knockdown or overexpression of CtBP2 in the ECA109 cells was confirmed using the observation of green fluorescence, reverse transcription-quantitative polymerase chain reaction (RT-qPCR) and western blotting.

$R N A$ extraction and $R T-q P C R$. Total RNA was extracted from the CtBP2 knockdown or overexpressing cells using TRIzol reagent (Thermo Fisher Scientific, Inc.) in accordance with the manufacturer's protocol. First-strand cDNA synthesis was conducted using a RevertAid First Strand cDNA Synthesis kit (Thermo Fisher Scientific, Inc.). The temperature protocol was $60 \mathrm{~min}$ at $42^{\circ} \mathrm{C}$, with termination of the reaction by heating at $70^{\circ} \mathrm{C}$ for 5 min. $\mathrm{qPCR}$ was performed in triplicate using SYBR Green Master mix (Roche Diagnostics, Basel, Switzerland) in a 7500 Real-Time PCR instrument (Applied Biosystems; Thermo Fisher Scientific, Inc.) to test the mRNA expression level of CtBP2. The thermocycling conditions were as follows: Firstly $95^{\circ} \mathrm{C}$ for $10 \mathrm{~min}$, followed by $95^{\circ} \mathrm{C}$ for $10 \mathrm{sec}, 60^{\circ} \mathrm{C}$ for $15 \mathrm{sec}$ and $72^{\circ} \mathrm{C}$ for $20 \mathrm{sec}$, for 40 cycles. The relative expression of mRNA was calculated using the $2^{-\Delta \Delta C q}$ method with GAPDH as an internal reference (30). The following primer sequences were used: GAPDH, 5'-GAC CTG ACC TGC CGT CTA-3' (sense) and 5'-AGG AGT GGG TGT CGC TGT-3' (antisense); CtBP2, 5'-CTG AGT TCC TGG CCT TTC TG-3' (sense) and 5'-GAC TTG ATA TCC GCG TCC TC-3' (antisense).

Western blot analysis. Briefly, cells were homogenized in lysis buffer containing $1 \mathrm{mM}$ phenylmethane sulfonyl fluoride and complete protein inhibitor mixture (Beyotime Institute of Biotechnology) for $15 \mathrm{~min}$ on ice, and then centrifuged at $13,400 \mathrm{x} \mathrm{g}$ for $10 \mathrm{~min}$ to collect the supernatant. The supernatant was diluted in $5 \mathrm{X}$ sodium dodecyl sulfate (SDS) loading buffer (Beyotime Institute of Biotechnology), boiled for $5 \mathrm{~min}$ and then cooled on ice. The protein concentration was measured at $280 \mathrm{~nm}$ using a One Drop Spectrophotometer (Wuyi Technology Co., Ltd., Nanjing, China) prior to loading the protein onto a gel (50 $\mu \mathrm{g}$ protein/lane). The proteins were separated by $10 \%$ SDS-PAGE and then blotted to polyvinylidene difluoride membranes (EMD Millipore, Billerica, MA, USA). The membrane was blocked with Tris-buffered saline and $0.1 \%$ Tween-20 (TBST) supplemented with 5\% non-fat milk for $2 \mathrm{~h}$ at room temperature, and then reacted with the following primary antibodies overnight at $4^{\circ} \mathrm{C}$ : Anti-CtBP2 (sc-17759; 1:200; Santa Cruz Biotechnology, Inc., Dallas, TX, USA), anti-activated caspase-3 (cleaved) (AB3623; 1:200; EMD Millipore), anti-B-cell lymphoma 2 (anti-Bcl-2; Ab-1; 1:1,000; EMD Millipore), anti-Bcl-2-associated X protein (anti-Bax; ab53154; 1:500; Abcam, Cambridge, MA, USA) and anti- $\beta$-actin (ab8227; $1: 1,000$; Abcam). After washing with TBST three times, the membrane was then reacted with horseradish peroxidase (HRP)-conjugated goat anti-rabbit IgG (A8919; 1:1,000) or HRP-conjugated rabbit anti-mouse IgG (A9044; 1,1000; both from Sigma-Aldrich; Merck KGaA, Darmstadt, Germany) for $2 \mathrm{~h}$ at $37^{\circ} \mathrm{C}$. The protein bands were 
A

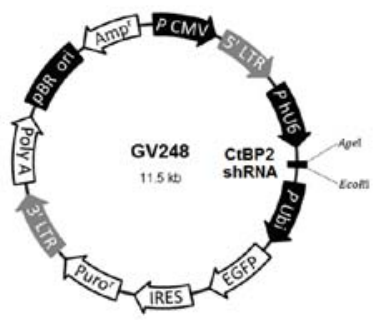

B

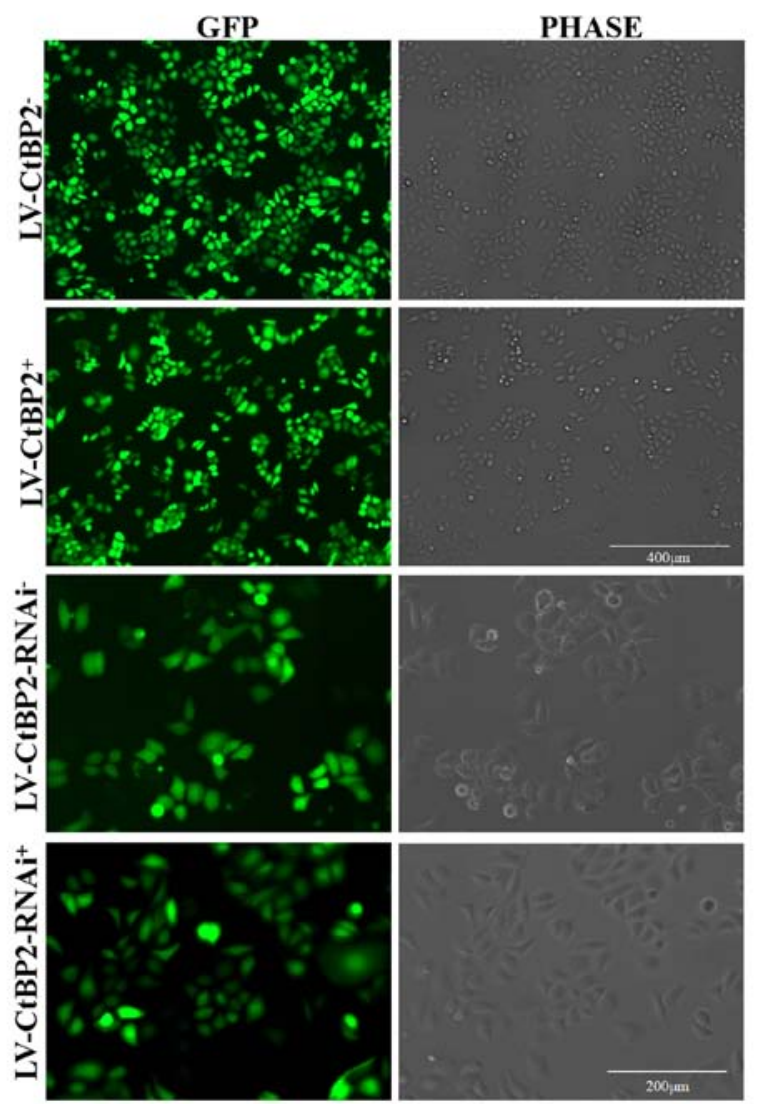

Figure 1. ECA109 cells were transfected with LV-CtBP2-RNAi or LV-CtBP2 for the knockdown or overexpression of $\mathrm{CtBP} 2$, respectively. (A) Diagrams showing the construction of the recombinant lentiviral vectors LV-CtBP2-RNAi (left) and LV-CtBP2 (right). (B) Following transfection, the ECA109 cells were observed using fluorescence and phase contrast microscopy. Scale bar, $400 \mu \mathrm{m}$ (upper pair of panel) and $200 \mu \mathrm{m}$ (lower pair of panels). LV-CtBP2-RNAi, lentivirus for CtBP2 knockdown via RNA interference; LV-CtBP2, lentivirus for CtBP2 overexpression; CtBP2, C-terminal binding protein-2; shRNA, small hairpin RNA; GFP, green fluorescence protein.

detected using ECL chemiluminescence reagent (Thermo Fisher Scientific, Inc.) and imaged using a chemiluminescence detection system (Tanon Science and Technology Co., Ltd., Shanghai, China). ImageJ software (National Institutes of Health, Bethesda, MD, USA) was used to analyze the density of the bands and $\beta$-actin was used as a reference for normalization. All experiments were repeated three times.

DDP treatment and cell viability assay. ECA109 cells were seeded in triplicate in a 96-well plate and cultured in $100 \mu \mathrm{l}$ DMEM medium containing $10 \%$ FCS. When the cells had become attached to the bottom of well, the cells were treated with DDP (Sigma-Aldrich; Merck KGaA) in serial dilutions (final concentration $1.5 \times 10^{-3}, 1.5 \times 10^{-4}, 1.5 \times 10^{-5}, 1.5 \times 10^{-6}$,
$1.5 \times 10^{-7}$ or $1.5 \times 10^{-8} \mathrm{M}$ ) in DMEM. Following incubation for $48 \mathrm{~h}$, methylthiazolyl tetrazolium solution (MTT; Beyotime Institute of Biotechnology; $10 \mu \mathrm{l} ; 5 \mathrm{mg} / \mathrm{ml}$ ) was added to each well and the plate was incubated for a further $4 \mathrm{~h}$. The medium was eliminated and $100 \mu \mathrm{l}$ dimethylsulfoxide (Merck KGaA) was added to each well. The plate was shaken to dissolve the MTT-formazan crystals and the absorbance at a wavelength of $570 \mathrm{~nm}$ was read using an ELX800 microplate reader (BioTek Instruments, Inc., Winooski, VT, USA).

On the basis of the MTT results for the various concentrations of DDP, the half maximal inhibitory concentration $\left(\mathrm{IC}_{50}\right)$ of DPP was determined. This concentration of DDP was subjected to further testing of cell viability with different treatment times $(0,12,24$ and $48 \mathrm{~h})$ in order to determine the appropriate treatment duration. For subsequent analysis of the role of CtBP2 in the cytotoxicity of DDP, transfected and untransfected ECA109 cells were exposed to the concentration of DPP closest to the $\mathrm{IC}_{50}$ using the treatment duration identified to be appropriate in this assay.

Hoechst 33342 staining. ECA109 cells on glass coverslips in 24 -well plates $\left(5 \times 10^{4}\right.$ cells/well) were fixed with $4 \%$ paraformaldehyde for $30 \mathrm{~min}$ at room temperature and then washed with $0.01 \%$ PBS three times, for $10 \mathrm{~min}$ each time at room temperature. The cells were then stained with Hoechst 33342 (Shanghai Yeasen Biotechnology Co., Ltd., Shanghai, China) for $10 \mathrm{~min}$ at room temperature. The slides were mounted with anti-fade solution (Beyotime Institute of Biotechnology). The apoptotic cells were identified by detecting the condensation and fragmentation of the cell nuclei under a fluorescence microscope (Zeiss AG, Oberkochen, Germany). Apoptotic cell numbers were counted in three randomly selected fields to calculate the apoptosis rate in triplicate.

Flow cytometry (FCM) assay. Following the aforementioned treatments, the cells were digested with trypsin and washed with $0.01 \%$ PBS twice. The cells were then stained for 10-20 min with Annexin V-fluorescein isothiocyanate and propidium iodide (Abcam) solution at room temperature for $1 \mathrm{~h}$ in the dark, and then subjected to FCM (BD Biosciences, San Jose, CA, USA). A total of 10,000 fluorescence signals of each group were collected. The data were analyzed using FACSuite software (BD Biosciences).

Statistical analysis. All data were analyzed using SPSS 17.0 software (SPSS, Inc., Chicago, IL, USA). One-way analysis of variance was used to analyze the data and Tukey's post hoc test was used to analyze the differences between specific groups. $\mathrm{P}<0.05$ was considered to indicate a statistically significant difference.

\section{Results}

Successful establishment of stable cell lines. In order to detect the impact of CtBP2 on DDP resistance in ESCC, the ECA109 cells were transfected with lentivirus to knockdown or overexpress $\mathrm{CtBP} 2$. As the recombinant lentiviral vector contained the enhanced green fluorescent protein (EGFP) gene, the rate of transfection could be determined by direct observation under a fluorescence microscope. The percentage 
A

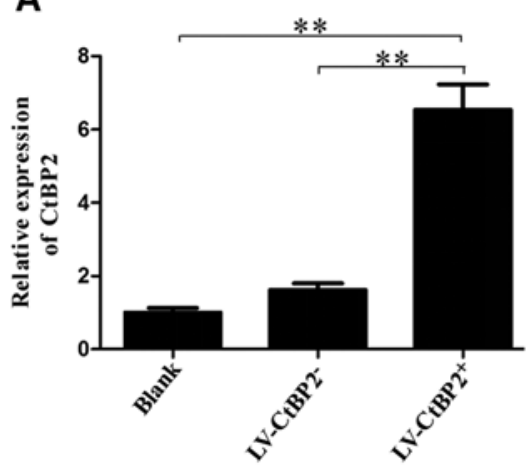

C

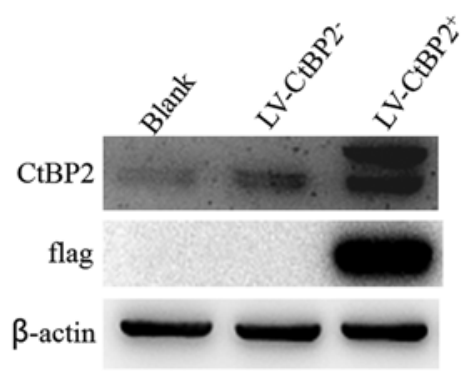

D

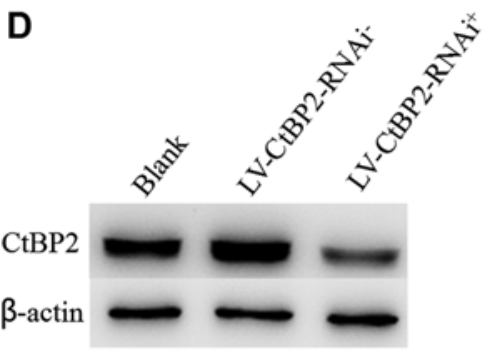

B
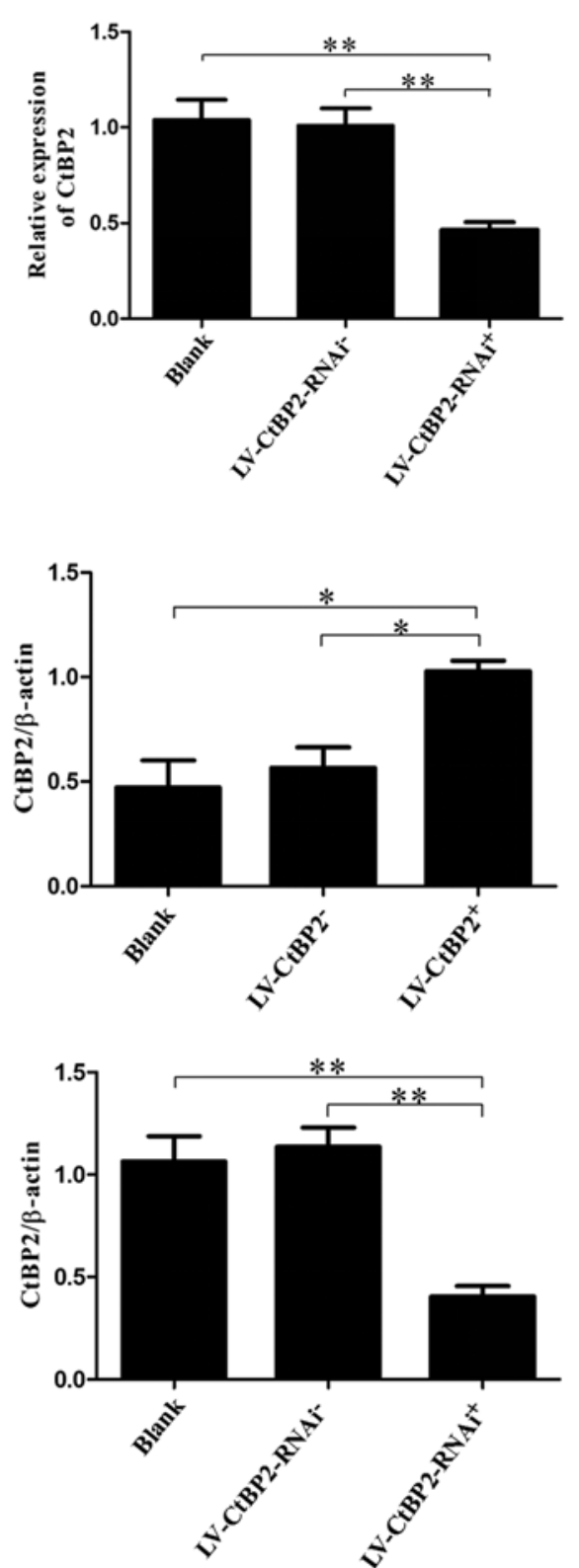

Figure 2. Relative expression levels of CtBP2 mRNA and protein in ECA109 cells were tested by RT-qPCR and western blotting. Relative levels of CtBP2 mRNA in ECA109 cells transfected with (A) LV-CtBP2 and (B) LV-CtBP2-RNAi were analyzed by RT-qPCR. The relative protein levels of CtBP2 in ECA109 cells transfected with (C) LV-CtBP2 and (D) LV-CtBP2-RNAi were analyzed by western blotting. ${ }^{*} \mathrm{P}<0.05$ and ${ }^{* *} \mathrm{P}<0.01$ as indicated. RT-qPCR, reverse transcription-quantitative polymerase chain reaction; blank, untransfected cells; LV-CtBP2-RNAi, lentivirus for CtBP2 knockdown via RNA interference; LV-CtBP2, lentivirus for CtBP2 overexpression; CtBP2, C-terminal binding protein-2.

of cells positive for EGFP was $>90 \%$ in the ECA109 cells transfected with recombinant LV-CtBP2-RNAi or LV-CtBP2. Fluorescence images of the transfected cells are presented in Fig. 1B.

The expression levels of CtBP2 in the ECA109 cells transfected with recombinant LV-CtBP2-RNAi or LV-CtBP2 (the LV-CtBP2-RNAi ${ }^{+}$and LV-CtBP2 ${ }^{+}$groups, respectively) were further confirmed by RT-qPCR and western blot analysis. The expression of CtBP2 mRNA was revealed to be significantly changed by RT-qPCR analysis. Compared with the blank and negative control (LV-CtBP2) groups, the relative expression of CtBP2 mRNA in the LV-CtBP2 $2^{+}$transfection group was increased $\sim 6$-fold $(\mathrm{P}<0.01)$. By contrast, the relative expression of CtBP2 mRNA in the LV-CtBP2-RNAi ${ }^{+}$ transfection group was decreased by more than half compared with that in the blank and negative control (LV-CtBP2-RNAi) groups $(\mathrm{P}<0.01)$. No significant difference in CtBP2 mRNA levels was detected between the blank group and the LV-CtBP2 or LV-CtBP2-RNAi group ( $\mathrm{P}>0.05$; Fig. $2 \mathrm{~A}$ and $\mathrm{B}$ ).

The protein levels of CtBP2 detected by western blotting exhibited similar trends to the expression levels of CtBP2 mRNA in the ECA109 cells subjected to CtBP2 knockdown or overexpression. Compared with the blank and respective negative control groups, the expression of $\mathrm{CtBP} 2$ was increased $\sim 2$-fold in the $\mathrm{LV}-\mathrm{CtBP} 2^{+}$transfection group $(\mathrm{P}<0.05)$ and decreased by two-thirds in the LV-CtBP2-RNAi ${ }^{+}$transfection 

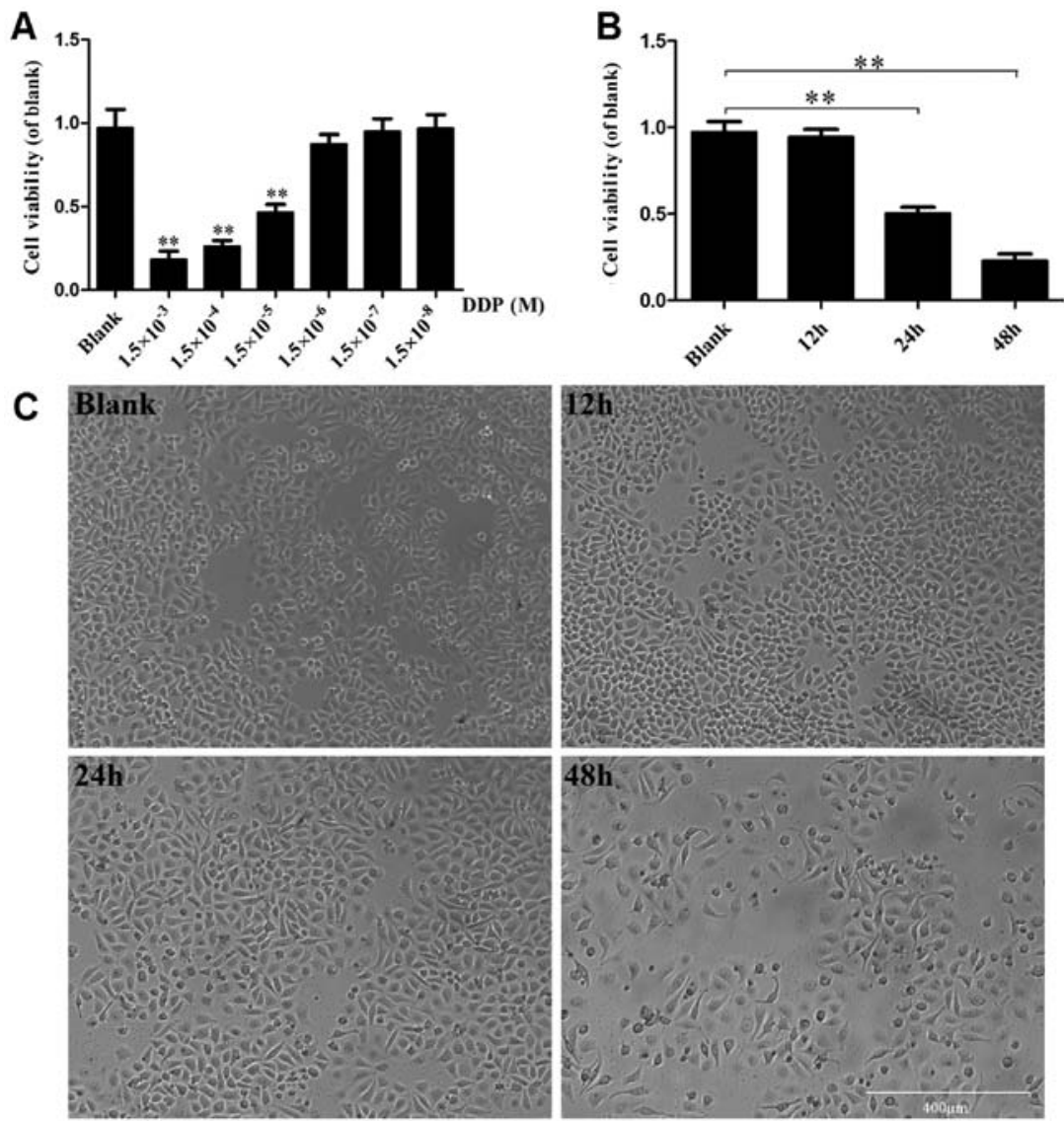

Figure 3. Cell viability was measured by MTT assay. The viability of ECA109 cells treated with (A) different concentrations of DDP for $48 \mathrm{~h}$ and (B) $1.5 \times 10^{-5} \mathrm{M}$ DDP for different time periods was analyzed by MTT assay. (C) ECA109 cells treated with $1.5 \times 10^{-5} \mathrm{M}$ DDP for different time periods were observed under an inverted phase contrast microscope (scale bar, $400 \mu \mathrm{m}$ ). ${ }^{* *} \mathrm{P}<0.01$ vs. blank. DDP, cisplatin; blank, cells without DDP treatment.

group $(\mathrm{P}<0.01$; Fig. $2 \mathrm{C}$ and $\mathrm{D})$. Furthermore, no significant difference was detected between the blank group and the LV-CtBP2- or LV-CtBP2-RNAi group ( $\mathrm{P}>0.05)$.

These results suggest that stable cell lines with the knockdown or overexpression of CtBP2 were successfully obtained following recombinant lentiviral transfection for use in the following experiments.

CtBP2 overexpression promotes ECA109 cell viability following DDP treatment. In order to determine the optimized concentration and treatment time for DDP, ECA109 cells were treated with DDP solution in serial dilutions for different time periods. Firstly, an MTT assay was conducted to test the changes in cell viability following treatment with serial dilutions of DDP. The cell viability was markedly decreased following treatment with increasing concentrations of DDP for $24 \mathrm{~h}$. Compared with the blank group, the cell viability in the $1.5 \times 10^{-3}, 1.5 \times 10^{-4}$ and $1.5 \times 10^{-5} \mathrm{M}$ DDP treated groups was significantly reduced $(\mathrm{P}<0.01)$. Notably, the cell viability of the $1.5 \times 10^{-5} \mathrm{M}$ DDP treatment group was reduced by $52.15 \%$ (Fig. 3A).

Secondly, the effects on cell viability of incubation with $1.5 \times 10^{-5} \mathrm{M}$ DDP for different treatment times were examined via MTT assay (Fig. 3B) and by observation under an inverted phase contrast microscope (Fig. 3C). When observed under the microscope, the number of dead cells appeared to increase gradually as the treatment time with DDP was prolonged. The MTT assay demonstrated that compared with the blank group, the viability of the cells treated with DDP for 24 and $48 \mathrm{~h}$ was significantly decreased $(\mathrm{P}<0.01)$, but the reduction in viability of the cells treated with DDP for $12 \mathrm{~h}$ was not significant ( $\mathrm{P}>0.05$; Fig. 3B).

These results indicate that the effect of DDP on cell viability was dependent on concentration and reaction time. The optimized concentration and treatment time for DDP in ECA109 cells were $1.5 \times 10^{-5} \mathrm{M}$ and $24 \mathrm{~h}$, respectively, and were used in the following experiments.

The impact of CtBP2 on the viability of the DDP-treated ECA109 cells was examined by microscopy and MTT assay (Fig. 4). The microscopy images revealed that cell shrinkage occurred following treatment with DDP, and the number of dead cells was increased. The viability of the ECA109 cells treated with DDP for $24 \mathrm{~h}$ was significantly reduced compared with that of the control cells $(\mathrm{P}<0.01)$. The cell viability of the LV-CtBP2-RNAi ${ }^{+}$+ DDP group was significantly reduced compared with that of the LV-CtBP2-RNAi + DDP group $(\mathrm{P}<0.05)$. Furthermore, the cell viability of the $\mathrm{LV}-\mathrm{CtBP} 2^{+}+$DDP group was significantly increased compared with that of the LV-CtBP2- + DDP group $(\mathrm{P}<0.01)$. No statistically significant difference was detected between the LV-CtBP2- + DDP and LV-CtBP2-RNAi + DDP groups and the blank + DDP group $(\mathrm{P}>0.05)$. These results indicate that the overexpression of CtBP2 attenuated the reduction of cell viability induced by DDP, and the knockdown of CtBP2 augmented the DDP-induced reduction of cell viability. 
A
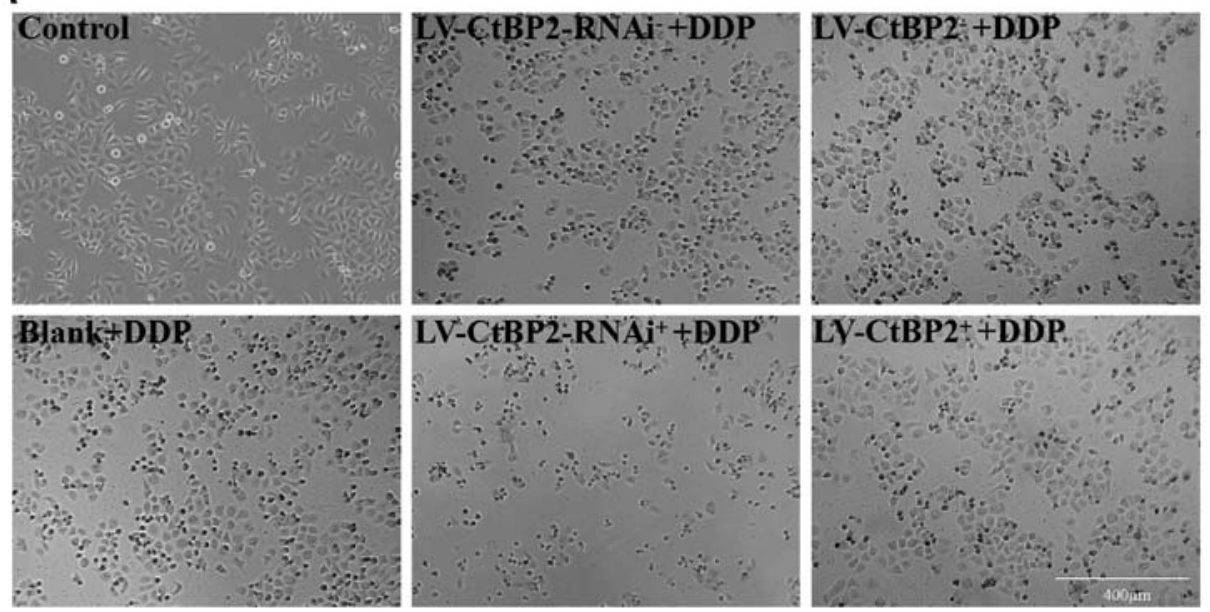

B

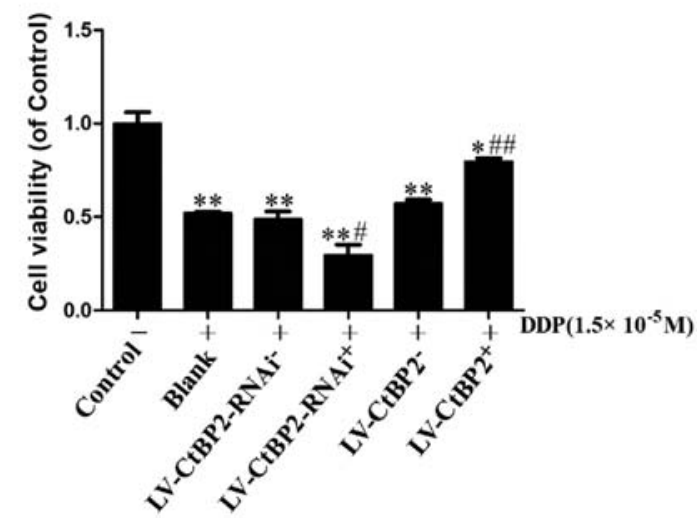

Figure 4. Effect of CtBP2 on the viability of ECA109 cells treated with DDP $\left(1.5 \times 10^{-5} \mathrm{M}\right)$ was evaluated. (A) Blank and transfected ECA109 cells treated with DPP were observed under an inverted phase contrast microscope (scale bar, $400 \mu \mathrm{m}$ ). (B) The viability of the ECA109 cells was analyzed by MTT assay. ${ }^{*} \mathrm{P}<0.05$ and ${ }^{* *} \mathrm{P}<0.01$ vs. control; ${ }^{*} \mathrm{P}<0.05$ and ${ }^{\# \#} \mathrm{P}<0.01$ vs. the blank control group (blank + DPP) or the respective negative control group (LV-CtBP2 + DPP or LV-CtBP2-RNAi + DPP). DDP, cisplatin; control, untreated control group; blank, untransfected cells; LV-CtBP2-RNAi, lentivirus for CtBP2 knockdown via RNA interference; LV-CtBP2, lentivirus for CtBP2 overexpression; CtBP2, C-terminal binding protein-2.

CtBP2 overexpression reduces the DDP-induced apoptosis of ECA109 cells. Hoechst 33342 staining and FCM were used to investigate the effect of CtBP2 on the cell apoptosis induced by DDP. The results of Hoechst 33342 staining demonstrated that the numbers of apoptotic bodies were significantly increased $(\mathrm{P}<0.01)$ in the DPP-treated cells compared with untreated control group. The number of apoptotic bodies was increased significantly in the LV-CtBP2-RNAi ${ }^{+}+$DDP group compared with the LV-CtBP2-RNAi + DDP group, and decreased significantly in the LV-CtBP2 ${ }^{+}+$DDP group compared with the LV-CtBP2- + DDP group $(\mathrm{P}<0.05$; Fig. 5A). On the basis of these results, it appears that CtBP2 overexpression attenuates the increase of apoptotic bodies induced by DDP.

FCM was used to further verify the impact of CtBP2 on the DDP-induced apoptosis of ECA109 cells. The results are consistent with those of Hoechst 33342 staining. The percentages of apoptotic cells in the untreated control, blank + DDP, LV-CtBP2-RNAi + DDP and LV-CtBP2-RNAi ${ }^{+}+$DDP groups were 5.34, 13.7, 13.98 and $21.59 \%$ respectively. Compared with the blank + DDP group and the LV-CtBP2-RNAi + DDP group, the percentage of apoptotic cells in the CtBP2 knockdown (LV-CtBP2-RNAi ${ }^{+}+$DDP) group was significantly increased $(\mathrm{P}<0.05)$. The percentages of apoptotic cells in the blank + DDP, LV-CtBP2 + DDP and LV-CtBP2 ${ }^{+}+$DDP groups were $13.7,14.75$ and $5.86 \%$ respectively. The percentage of apoptotic cells in the CtBP2 overexpression $\left(\mathrm{LV}-\mathrm{CtBP} 2^{+}+\mathrm{DDP}\right)$ group was significantly lower than those of the LV-CtBP2 + DDP and blank + DDP groups $(\mathrm{P}<0.05$; Fig. 5B). The FCM results further indicate that CtBP2 overexpression inhibited the DDP-induced apoptosis of ECA109 cells.

Mechanisms underlying the effect of CtBP2 on DDP chemoresistance. Caspase serves essential roles in cell apoptosis, which is a cellular event considered as programmed cell death $(31,32)$. Caspase- 3 is one of the crucial downstream effectors of apoptosis (33). The effects of CtBP2 on the protein levels of p53, Bcl-2, Bax and activated caspase-3 were analyzed by western blotting (Fig. 6). The results shown in Fig. 6A and $\mathrm{B}$ demonstrate that the expression of p53 was significantly increased $(\mathrm{P}<0.01)$ in the ECA109 cells treated with $1.5 \times 10^{-5} \mathrm{M}$ DDP for $24 \mathrm{~h}$ compared with the untreated control cells. Notably, the expression of p53 in the CtBP2 overexpression $\left(\mathrm{LV}-\mathrm{CtBP} 2^{+}+\mathrm{DDP}\right)$ group was significantly decreased compared with that of the LV-CtBP2 + DDP group $(\mathrm{P}<0.05)$. The changes in cleaved caspase-3 levels (Fig. 6D) exhibited a similar pattern to those of $\mathrm{p} 53$. These results demonstrate 
A
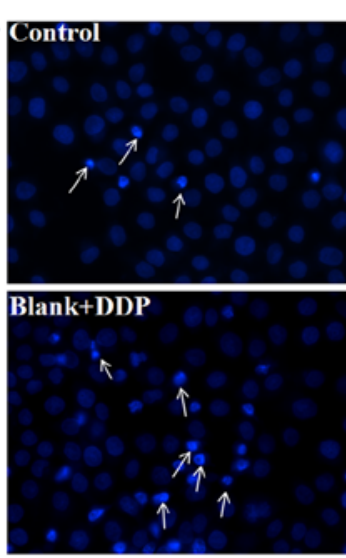

B

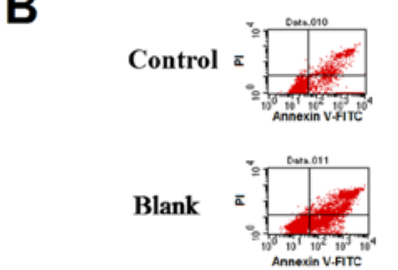

LV-CtBP2-RNAi ${ }^{-}$
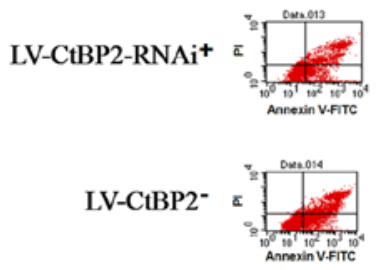

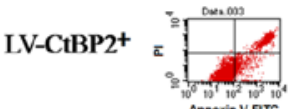
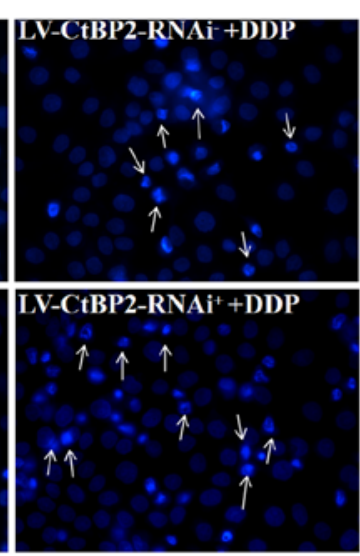
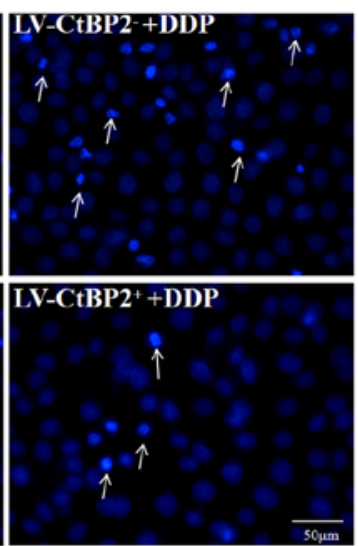
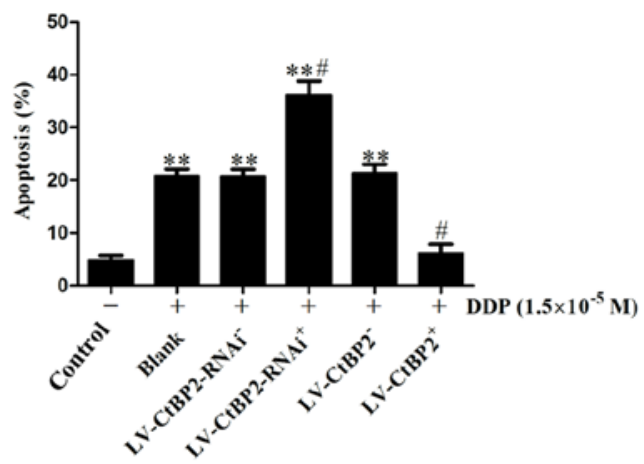

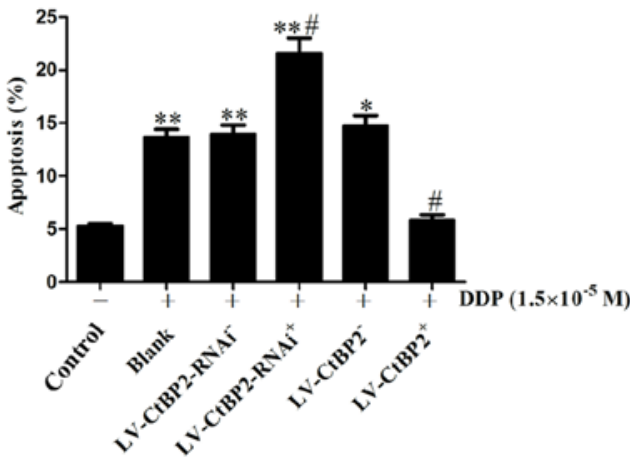

Figure 5. Effect of CtBP2 on the apoptosis of ECA109 cells treated with DDP $\left(1.5 \times 10^{-5} \mathrm{M}\right)$ was detected by Hoechst 33342 staining and FCM. (A) Hoechst 33342 staining and quantitative analysis of the results. In the fluorescence microscopy images, apoptotic bodies are indicated by arrows (scale bar, $50 \mu \mathrm{m})$. (B) The apoptosis of ECA109 cells was analyzed by FCM assay. ${ }^{* *} \mathrm{P}<0.01$ vs. control; ${ }^{\#} \mathrm{P}<0.05$ vs. the blank control group (blank + DPP) or the respective negative control group (LV-CtBP2- + DPP or LV-CtBP2-RNAi + DPP). DDP, cisplatin; control, untreated control group; blank, untransfected cells; LV-CtBP2-RNAi, lentivirus for CtBP2 knockdown via RNA interference; LV-CtBP2, lentivirus for CtBP2 overexpression; CtBP2, C-terminal binding protein-2; FCM, flow cytometry; PI, propidium iodide; FITC, fluorescein isothiocyanate.

that the overexpression of CtBP2 inhibited the DDP-induced increase in the protein levels of p53 and activated caspase- 3 in ECA109 cells.

The protein levels of Bax and Bcl-2 were also detected using western blotting. The results clearly demonstrate that Bcl-2 expression was downregulated while Bax expression was upregulated in the $\mathrm{CtBP} 2$ knockdown (LV-CtBP2-RNAi ${ }^{+}+$DDP) group. Therefore, the Bcl-2/Bax ratio in the $\mathrm{LV}-\mathrm{CtBP} 2-\mathrm{RNAi}^{+}+\mathrm{DDP}$ group was significantly decreased compared with those in the untreated control, blank + DDP and LV-CtBP2-RNAi + DDP groups $(\mathrm{P}<0.05)$. By contrast, the ratio of $\mathrm{Bcl}-2 / \mathrm{Bax}$ in the $\mathrm{CtBP} 2$ overexpression (LV-CtBP2 $2^{+}+$DDP) group was significantly increased compared with those in the blank + DDP and LV-CtBP2 ${ }^{+}+\mathrm{DDP}$ groups $(\mathrm{P}<0.05$; Fig. $6 \mathrm{C})$. These results indicate that $\mathrm{CtBP} 2$ overexpression alleviated the DDP-induced apoptosis of
ECA109 cells by reducing the protein levels of p53, cleaved caspase- 3 and Bax, and increasing Bcl-2 expression.

\section{Discussion}

Understanding of the biology and molecular mechanisms underlying ESCC development and progression is required for advances in the treatment of ESCC (34). Chemotherapy is one of the main therapeutic strategies for the treatment of ESCC $(35,36)$. However, chemoresistance to anticancer drugs greatly reduces the effectiveness of these drugs, and is a huge obstacle to the discovery of a successful therapy for ESCC (37). Therefore, it is urgently necessary to identify an effective potential therapeutic target for combatting drug resistance in ESCC. DDP is a first-line drug in the treatment for ESCC, and DDP resistance remains a serious challenge. 
A

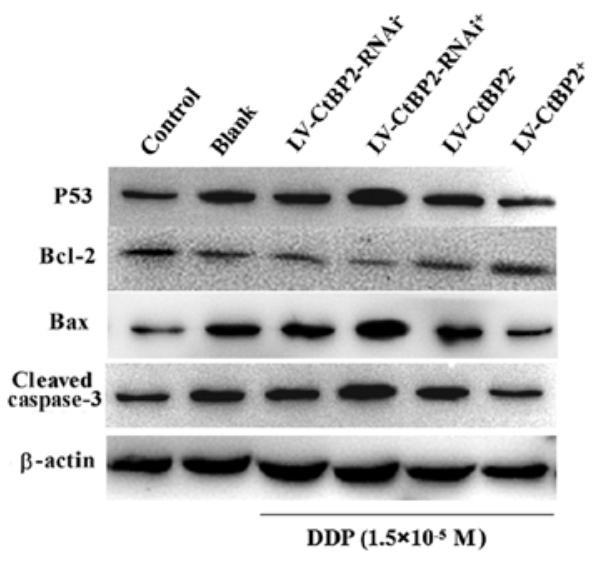

B

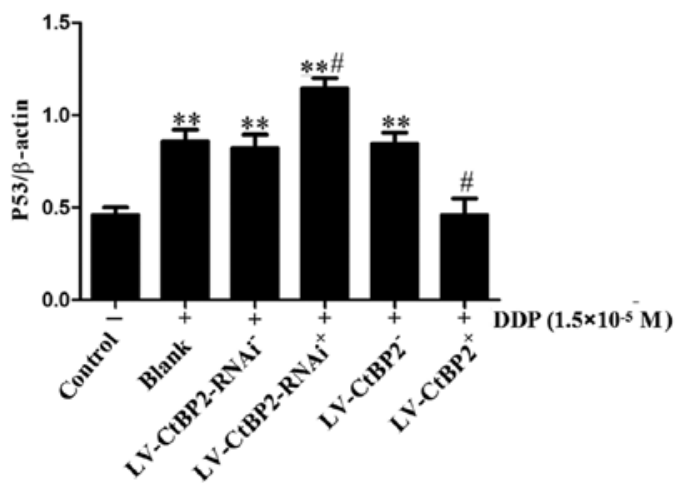

C

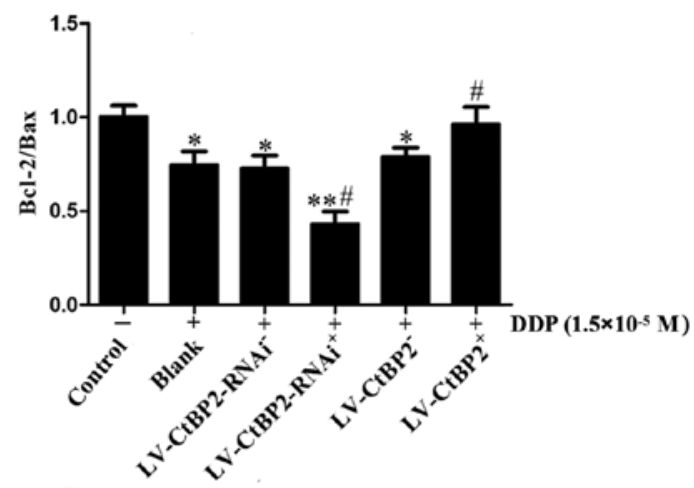

D

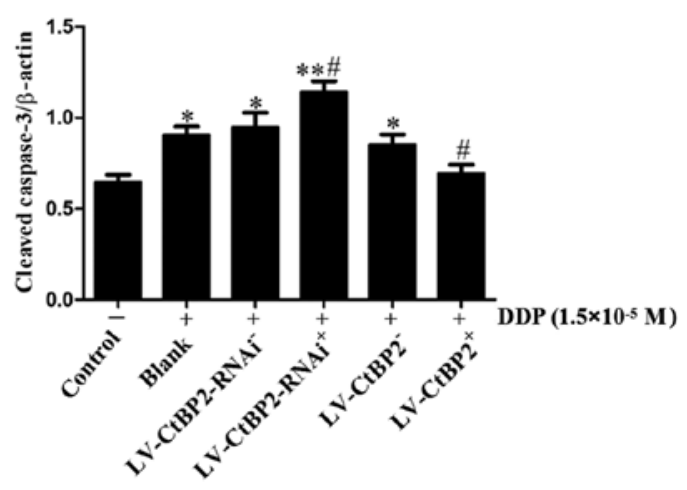

Figure 6. Levels of apoptosis-associated proteins, namely p53, Bcl-2, Bax and cleaved caspase-3, were measured by western blotting. (A) Representative western blots for p53, Bcl-2, Bax and cleaved caspase-3. Quantified data for (B) p53, (C) the ratio of Bcl-2/Bax and (D) cleaved caspase-3. ${ }^{*}<0.05$ and ${ }^{* *} \mathrm{P}<0.01$ vs. control; " $\mathrm{P}<0.05$ vs. blank control group (blank + DPP) or the respective negative control group (LV-CtBP2 + DPP or LV-CtBP2-RNAi + DPP). DDP, cisplatin; control, untreated control group; blank, untransfected cells; LV-CtBP2-RNAi, cells transfected with a lentivirus for CtBP2 knockdown; LV-CtBP2, cells transfected with a lentivirus for CtBP2 overexpression; CtBP2, C-terminal binding protein-2; Bcl-2, B-cell lymphoma 2; Bax, Bcl-2-associated X protein.

A previous study conducted by the present research team indicated that the expression of CtBP2 was upregulated in ESCC tissues compared with adjacent non-tumorous tissues (17). However, the effect of CtBP2 on the susceptibility of ESCC cells to DDP was unclear. Therefore, the present study established stable ECA109 cells with the overexpression or knockdown of CtBP2 via recombinant lentiviral transfection in order to investigate the effect of $\mathrm{CtBP} 2$ on these cells when treated with DDP.

In the present study, the optimized concentration and treatment time of DDP for use in vitro were determined by MTT assay; $1.5 \times 10^{-5} \mathrm{M}$ DDP treatment for $24 \mathrm{~h}$ was selected for further investigation. The optimized concentration $\left(1.5 \times 10^{-5} \mathrm{M}\right)$ in the study is similar to the clinically used dose of DDP (20 mg/m²/day) $(38,39)$. The effect of CtBP2 on the cell apoptosis induced by DDP was investigated by Hoechst 33342 staining and FCM. The overexpression of CtBP2 attenuated the reduction of cell viability and inhibited the cell apoptosis induced by DDP. By comparison, the knockdown of CtBP2 increased the susceptibility of ECA109 cells to DDP, as it increased the number of apoptotic cells following treatment with DDP. These findings indicate that CtBP2 is a potential target for the chemotherapy of ESCC, via which drug resistance may be reversed in patients with ESCC.

There are various mechanisms by which tumor cells develop resistance to chemotherapy. CtBP2 has been demonstrated to reduce the chemosensitivity of breast cancer cells to various chemotherapeutic drugs via p53-dependent and p53-independent effects (29). The present study attempted to further investigate the effect of $\mathrm{CtBP} 2$ on the chemoresistance of ECA109 cells. The protein levels of the apoptosis-related proteins $\mathrm{p} 53, \mathrm{Bcl}-2$, Bax and cleaved caspase-3 in the DDP-treated ECA109 cells with CtBP2 overexpression or knockdown were determined using western blotting. The overexpression of $\mathrm{CtBP} 2$ reduced the protein levels of $\mathrm{p} 53$, cleaved caspase- 3 and Bax, and increased the protein levels of Bcl-2 in DDP-treated ECA109 cells, and the knockdown of CtBP2 exhibited opposing effects. These results indicated that CtBP2 reduced the chemosensitivity of ECA109 cells to DPP via the inhibition of $\mathrm{p} 53$, caspase-3 and Bax. This information supplements the findings of a previous study by the present research team, which demonstrated that $\mathrm{CtBP} 2$ promotes the progression of ESCC via the negative transcriptional regulation of p16 ${ }^{\mathrm{INK} 4 \mathrm{~A}}(27)$. Nuclear $\mathrm{p} 16$ has been reported to be important to the chemosensitivity of multiple cancers $(40,41)$, and in ESCC, CtBP2 promotes chemoresistance through the negative transcriptional regulation of $\mathrm{p} 16^{\mathrm{INK} 4 \mathrm{~A}}$. However, a limitation of the present study is that only a single cell line was investigated, and the further investigation of other cell types of esophageal cancer is required to confirm the findings of the study.

In conclusion, the results of the present study indicate that CtBP2 attenuated the susceptibility of ESCC cells to DDP by 
regulating the expression of apoptosis-related proteins and thereby inhibiting cell apoptosis. This knowledge may provide a new strategy for decreasing the chemoresistance to DDP in the treatment of ESCC.

\section{Acknowledgements}

Not applicable.

\section{Funding}

The present study was supported by grants from the National Natural Science Foundation of China (grant nos. 81101159 and 81502055 ), and the Natural Science Foundation of Jiangsu Province, China (grant no. BK20151268).

\section{Availability of data and materials}

The analyzed data sets generated during the study are available from the corresponding author on reasonable request.

\section{Authors' contributions}

MJ, YZ, HS, YM and QJ designed and did the research. QJ, YW, WB and PW analyzed the data. All authors have read and approved the final manuscript.

\section{Ethics approval and consent to participate}

Not applicable.

\section{Consent for publication}

Not applicable.

\section{Competing interests}

The authors declare that they have no competing interests with respect to the data appearing in the manuscript.

\section{References}

1. Wakatsuki K, Matsumoto S, Migita K, Ito M, Kunishige T, Nakade H, Nakatani M, Kitano M, Takano M, Obayashi C, et al: Usefulness of computed tomography density of a tumor in predicting the response of advanced esophageal cancer to preoperative chemotherapy. Surgery 162: 823-835, 2017.

2. Servagi-Vernat S, Créhange G, Bonnetain F, Mertens C, Brain E and Bosset JF: Chemoradiation in elderly esophageal cancer patients: Rationale and design of a phase I/II multicenter study (OSAGE). BMC Cancer 17: 483, 2017.

3. Canto MI, Abrams J, Kunzli H, Weusten B, Komatsu Y, Jobe BA and Lightdale CJ: Nitrous oxide cryotherapy for treatment of esophageal squamous cell neoplasia: Initial multicenter international experience with a novel portable cryoballoon ablation system (with video). Gastrointest Endosc 87: 574-581, 2018.

4. Pennathur A, Gibson MK, Jobe BA and Luketich JD: Oesophageal carcinoma. Lancet 381: 400-412, 2013.

5. Li Y, Wu X, Li L, Liu Y, Xu C, Su D and Liu Z: The E3 ligase HECTD3 promotes esophageal squamous cell carcinoma (ESCC) growth and cell survival through targeting and inhibiting caspase-9 activation. Cancer Lett 404: 44-52, 2017.

6. Kjaer DW, Larsson H, Svendsen LB and Jensen LS: Changes in treatment and outcome of oesophageal cancer in Denmark between 2004 and 2013. Br J Surg 104: 1338-1345, 2017.
7. Martin-Richard M, Díaz Beveridge R, Arrazubi V, Alsina M, Galan Guzmán M, Custodio AB, Gómez C, Muñoz FL, Pazo R and Rivera F: SEOM Clinical Guideline for the diagnosis and treatment of esophageal cancer (2016). Clin Transl Oncol 18: 1179-1186, 2016.

8. Sohda M and Kuwano H: Current status and future prospects for esophageal cancer treatment. Ann Thorac Cardiovasc Surg 23: 1-11, 2017.

9. Arnold M, Laversanne M, Brown LM, Devesa SS and Bray F: Predicting the future burden of esophageal cancer by histological subtype: International trends in incidence up to 2030. Am J Gastroenterol 112: 1247-1255, 2017.

10. Palumbo Júnior A, Da Costa NM, Esposito F, Fusco A and Pinto LF: High Mobility Group A proteins in esophageal carcinomas. Cell Cycle 15: 2410-2413, 2016.

11. Pan F, Mao H, Bu F, Tong X, Li J, Zhang S, Liu X, Wang L, Wu L, Chen R, et al: Sp1-mediated transcriptional activation of miR-205 promotes radioresistance in esophageal squamous cell carcinoma. Oncotarget 8: 5735-5752, 2017.

12. Sugihara H, Ishimoto T, Miyake K, Izumi D, Baba Y, Yoshida N, Watanabe $\mathrm{M}$ and Baba $\mathrm{H}$ : Noncoding RNA expression aberration is associated with cancer progression and is a potential biomarker in esophageal aquamous cell carcinoma. Int $\mathbf{J}$ Mol Sci 16: 27824-27834, 2015.

13. Goense L, van Rossum PS, Kandioler D, Ruurda JP, Goh KL, Luyer MD, Krasna MJ and van Hillegersberg R: Stage-directed individualized therapy in esophageal cancer. Ann N Y Acad Sci 1381: 50-65, 2016

14. Cleary JM, Mamon HJ, Szymonifka J, Bueno R, Choi N, Donahue DM, Fidias PM, Gaissert HA, Jaklitsch MT, Kulke MH, et al: Neoadjuvant irinotecan, cisplatin, and concurrent radiation therapy with celecoxib for patients with locally advanced esophageal cancer. BMC Cancer 16: 468, 2016.

15. Phatak P, Byrnes KA, Mansour D, Liu L, Cao S, Li R, Rao JN, Turner DJ, Wang JY and Donahue JM: Overexpression of miR-214-3p in esophageal squamous cancer cells enhances sensitivity to cisplatin by targeting survivin directly and indirectly through CUG-BP1. Oncogene 35: 2087-2097, 2016.

16. $\mathrm{Wu} \mathrm{Y}$ and Jiang M: The revolution of lung cancer treatment: from vaccines, to immune checkpoint inhibitors, to chimeric antigen receptor T therapy. Biotarget 1: 7, 2017.

17. Liu T, Li R, Zhao H, Deng J, Long Y, Shuai MT, Li Q, Gu H, Chen YQ and Leng AM: eIF4E promotes tumorigenesis and modulates chemosensitivity to cisplatin in esophageal squamous cell carcinoma. Oncotarget 7: 66851-66864, 2016.

18. Komatsu S, Ichikawa D, Kawaguchi T, Miyamae M, Okajima W, Ohashi T, Imamura T, Kiuchi J, Konishi H, Shiozaki A, et al: Circulating miR-21 as an independent predictive biomarker for chemoresistance in esophageal squamous cell carcinoma. Am J Cancer Res 6: 1511-1523, 2016.

19. Sumner ET, Chawla AT, Cororaton AD, Koblinski JE, Kovi RC, Love IM, Szomju BB, Korwar S, Ellis KC and Grossman SR: Transforming activity and therapeutic targeting of C-terminalbinding protein 2 in Apc-mutated neoplasia. Oncogene 36: 4810-4816, 2017.

20. Dai F, Xuan Y, Jin JJ, Yu S, Long ZW, Cai H, Liu XW, Zhou Y, Wang YN, Chen Z, et al: CtBP2 overexpression promotes tumor cell proliferation and invasion in gastric cancer and is associated with poor prognosis. Oncotarget 8: 28736-28749, 2017.

21. Riku M, Inaguma S, Ito $H$, Tsunoda $T$, Ikeda $H$ and Kasai $K$ : Down-regulation of the zinc-finger homeobox protein TSHZ2 releases GLI1 from the nuclear repressor complex to restore its transcriptional activity during mammary tumorigenesis. Oncotarget 7: 5690-5701, 2016.

22. Zheng X, Song T, Dou C, Jia Y and Liu Q: CtBP2 is an independent prognostic marker that promotes GLI1 induced epithelial-mesenchymal transition in hepatocellular carcinoma. Oncotarget 6: 3752-3769, 2015

23. Zhang C, Li S, Qiao B, Yang K, Liu R, Ma B, Liu Y, Zhang Z and $\mathrm{Xu}$ Y: CtBP2 overexpression is associated with tumorigenesis and poor clinical outcome of prostate cancer. Arch Med Sci 11: 1318-1323, 2015.

24. Yang X, Sun Y, Li H, Shao Y, Zhao D, Yu W and Fu J: C-terminal binding protein-2 promotes cell proliferation and migration in breast cancer via suppression of p16INK4A. Oncotarget 8: 26154-26168, 2017.

25. Frietze S, O'Geen H, Littlepage LE, Simion C, Sweeney CA, Farnham PJ and Krig SR: Global analysis of ZNF217 chromatin occupancy in the breast cancer cell genome reveals an association with ERalpha. BMC Genomics 15: 520, 2014. 
26. May T, Yang J, Shoni M, Liu S, He H, Gali R, Ng SK, Crum C, Berkowitz RS and Ng SW: BRCA1 expression is epigenetically repressed in sporadic ovarian cancer cells by overexpression of C-terminal binding protein 2. Neoplasia 15: 600-608, 2013.

27. Guan C, Shi H, Wang H, Zhang J, Ni W, Chen B, Hou S, Yang X, Shen A and Ni R: CtBP2 contributes to malignant development of human esophageal squamous cell carcinoma by regulation of p16INK4A. J Cell Biochem 114: 1343-1354, 2013.

28. Zhang J, Zhu J, Yang L, Guan C, Ni R, Wang Y, Ji L and Tian Y: Interaction with $\mathrm{CCNH} / \mathrm{CDK} 7$ facilitates $\mathrm{CtBP} 2$ promoting esophageal squamous cell carcinoma (ESCC) metastasis via upregulating epithelial-mesenchymal transition (EMT) progression. Tumour Biol 36: 6701-6714, 2015.

29. Birts CN, Harding R, Soosaipillai G, Halder T, Azim-Araghi A, Darley M, Cutress RI, Bateman AC and Blaydes JP: Expression of CtBP family protein isoforms in breast cancer and their role in chemoresistance. Biol Cell 103: 1-19, 2010.

30. Livak KJ and Schmittgen TD: Analysis of relative gene expression data using real-time quantitative PCR and the 2(-Delta Delta C(T)) Method. Methods 25: 402-408, 2001.

31. Ansari S, Chen C, Hasani-Sadrabadi MM, Yu B, Zadeh HH, $\mathrm{Wu} \mathrm{BM}$ and Moshaverinia A: Hydrogel elasticity and microarchitecture regulate dental-derived mesenchymal stem cell-host immune system cross-talk. Acta Biomater 60: 181-189, 2017.

32. Mihaly SR, Sakamachi Y, Ninomiya-Tsuji J and Morioka S: Noncanocial cell death program independent of caspase activation cascade and necroptotic modules is elicited by loss of TGF $\beta$-activated kinase 1. Sci Rep 7: 2918, 2017.

33. Luna C, Mendoza N, Casao A, Pérez-Pé R, Cebrián-Pérez JA and Muiño-Blanco T: c-Jun N-terminal kinase and p38 mitogenactivated protein kinase pathways link capacitation with apoptosis and seminal plasma proteins protect sperm by interfering with both routes. Biol Reprod 96: 800-815, 2017.

34. Whiteside TL: Stimulatory role of exosomes in the context of therapeutic anti-cancer vaccines. Biotarget 1: 5, 2017.
35. Kwon D, Yun JY, Keam B, Kim YT and Jeon YK: Prognostic implications ofFGFR1andMYCstatus in esophageal squamous cell carcinoma. World J Gastroenterol 22: 9803-9812, 2016.

36. Chen P, Zhang JY, Sha BB, Ma YE, Hu T, Ma YC, Sun H, Shi JX, Dong ZM and Li P: Luteolin inhibits cell proliferation and induces cell apoptosis via down-regulation of mitochondrial membrane potential in esophageal carcinoma cells EC1 and KYSE450. Oncotarget 8: 27471-27480, 2017.

37. Zhang HF, Wu C, Alshareef A, Gupta N, Zhao Q, Xu XE, Jiao JW, Li EM, Xu LY and Lai R: The PI3K/AKT/c-MYC axis promotes the acquisition of cancer stem-like features in esophageal squamous cell carcinoma. Stem Cells 34: 2040-2051, 2016.

38. Konishi H, Fujiwara H, Shiozaki A, Shoda K, Kosuga T, Kubota T, Okamoto K and Otsuji E: Effects of neoadjuvant 5 -fluorouracil and cisplatin therapy in patients with clinical stage II/III esophageal squamous cell carcinoma. Anticancer Res 38: 1017-1023, 2018 .

39. Liu B, Wang C, Chen P, Cheng B and Cheng Y: RACKI induces chemotherapy resistance in esophageal carcinoma by upregulating the PI3K/AKT pathway and Bcl-2 expression. Onco Targets Ther 11: 211-220, 2018.

40. Veena MS, Wilken R, Zheng JY, Gholkar A, Venkatesan N, Vira D, Ahmed S, Basak SK, Dalgard CL, Ravichandran S, et al: p16 protein and gigaxonin are associated with the ubiquitination of $\mathrm{NF}_{\kappa} \mathrm{B}$ in cisplatin-induced senescence of cancer cells. J Biol Chem 289: 34921-34937, 2014.

41. Geiger JL, Lazim AF, Walsh FJ, Foote RL, Moore EJ, Okuno SH, Olsen KD, Kasperbauer JL, Price DL, Garces YI, et al: Adjuvant chemoradiation therapy with high-dose versus weekly cisplatin for resected, locally-advanced HPV/p16-positive and negative head and neck squamous cell carcinoma. Oral Oncol 50: 311-318, 2014. 